\title{
Intraoperative diagnosis with abnormal branching of the left A8 pulmonary artery from the left main pulmonary artery
}

\author{
Yasushi Mizukami* ${ }^{*}$, Nobuhito Ueda and Hirofumi Adachi
}

\begin{abstract}
Background: Safety is of vital importance for lung resection. The dissection of pulmonary vessels is associated with vascular injury and bleeding, and identification of the vessels is necessary. The most common abnormal branching pattern of the left pulmonary artery is the mediastinal lingular artery. However, a mediastinal basal pulmonary artery is very rare. A case of abnormal branching from the left pulmonary artery to $\mathbf{S} 8$ which was diagnosed intraoperatively, and, thus, its dissection was avoided, is reported.

Case presentation: A 76-year-old woman with rheumatoid arthritis was diagnosed with left upper lung adenocarcinoma and visited our hospital. Contrast CT was not performed due to renal dysfunction, and abnormal branching of the left pulmonary artery was not identified. Video-assisted thoracoscopic left upper lobectomy and lymphadenectomy were performed. After the upper pulmonary vein was dissected and tissue around it was detached carefully, a pulmonary mediastinal branch from the left main pulmonary artery was identified descending between the upper pulmonary vein and upper bronchus. It was possible to separate the interlobar fissure safely and preserve A8. On retrospective examination, non-contrast $C T$ showed A8.
\end{abstract}

Conclusions: Although preoperative identification of left pulmonary mediastinal branches was difficult by non-contrast $\mathrm{CT}$, a careful surgical procedure preserved the left pulmonary mediastinal A8.

Keywords: Left pulmonary abnormal branch, Mediastinal basal pulmonary artery to S8, Lung cancer

\section{Background}

In lung resection, safety is vitally important. In particular, dissection of pulmonary vessels is associated with vascular injury and bleeding, and identification of the vessels is necessary. The most common abnormal branching pattern of the left pulmonary artery is the mediastinal lingular artery. However, a mediastinal basal pulmonary artery is very rare. A case of abnormal branching from the left pulmonary artery to S8 that was diagnosed intraoperatively and its dissection was avoided is reported.

\section{Case presentation}

The patient was a 76-year-old woman with rheumatoid arthritis. Computed tomography (CT) had been

\footnotetext{
* Correspondence: mizukam@gmail.com

Department of Thoracic Surgery, National Hospital Organization, Hokkaido

Cancer Center, 2-3-54 Kikusui 4-jo, Shiroishi-ku, Sapporo-shi, Hokkaido 003-0804, Japan
}

performed for a medical checkup, and a small nodule was detected in the left upper lobe $(\mathrm{S} 1+2)$ a year before she visited our hospital. Follow-up CT showed that the nodule with indentation was growing to $22 \mathrm{~mm}$ in size, and she was referred to a nearby hospital (Fig. 1). Fluorodeoxyglucose (FDG)-positron emission tomography (PET), brain magnetic resonance imaging, and transbronchial biopsy were performed, showing left upper lung adenocarcinoma classified as cT1bNOM0 Stage IA according to the Union for International Cancer Control classification (seventh edition). Though contrast-enhanced three-dimensional computed tomographic angiography (3DCT) of the pulmonary vessels was usually performed to identify the pulmonary branches preoperatively at that time, it was not performed in the present case due to renal dysfunction, and abnormal branching of the left pulmonary artery was not identified. Video-assisted thoracoscopic left upper lobectomy and lymphadenectomy were performed. $\mathrm{A} 1+2 \mathrm{c}$, the upper pulmonary vein, mediastinal $\mathrm{A} 4+5$, 


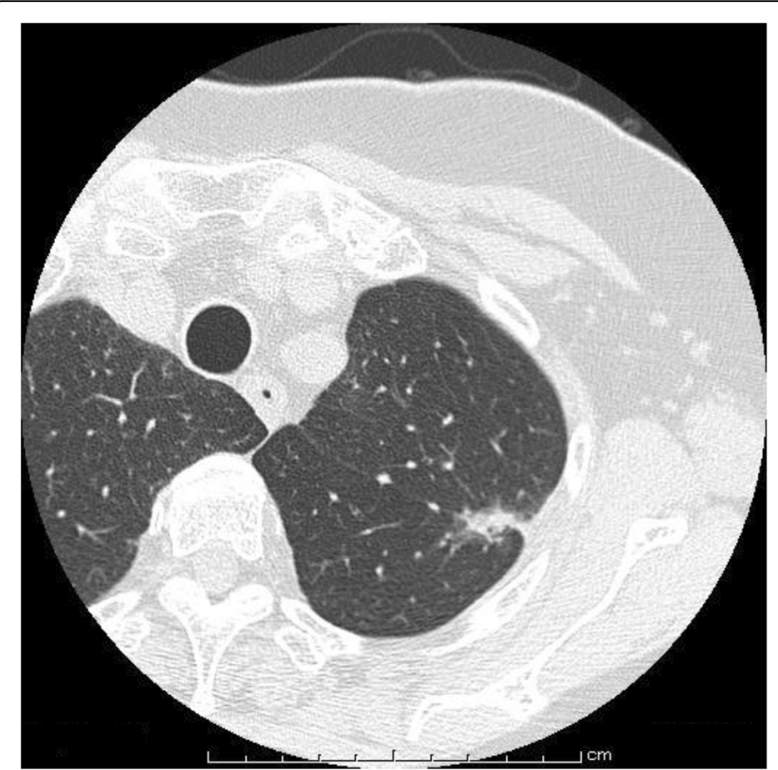

Fig. 1 Chest-computed tomography findings. It shows the nodule of left $\mathrm{S} 1+2$ with indentation. It is $22 \mathrm{~mm}$ in size

$\mathrm{A} 3$, and $\mathrm{A} 1+2 \mathrm{a}+\mathrm{b}$ were detached carefully and dissected. Before separation of the interlobar fissure was completed, a pulmonary mediastinal branch from the left main pulmonary artery was identified descending between the upper pulmonary vein and upper bronchus. The interlobar fissure was separated safely with preservation of A8 (Figs. 2 and 3). On retrospective examination, non-contrast CT showed A8 (Fig. 4).

\section{Conclusions}

An abnormal pulmonary branch can induce unexpected bleeding, and careful surgery is needed to dissect the branch to the preserved lobe. The number of left pulmonary branches varies from two to seven, and variations may occur in all segments. Generally, the first anterior branch supplies the lingular division in less than $10 \%$ of cases [1]. Recently, lingular artery branching

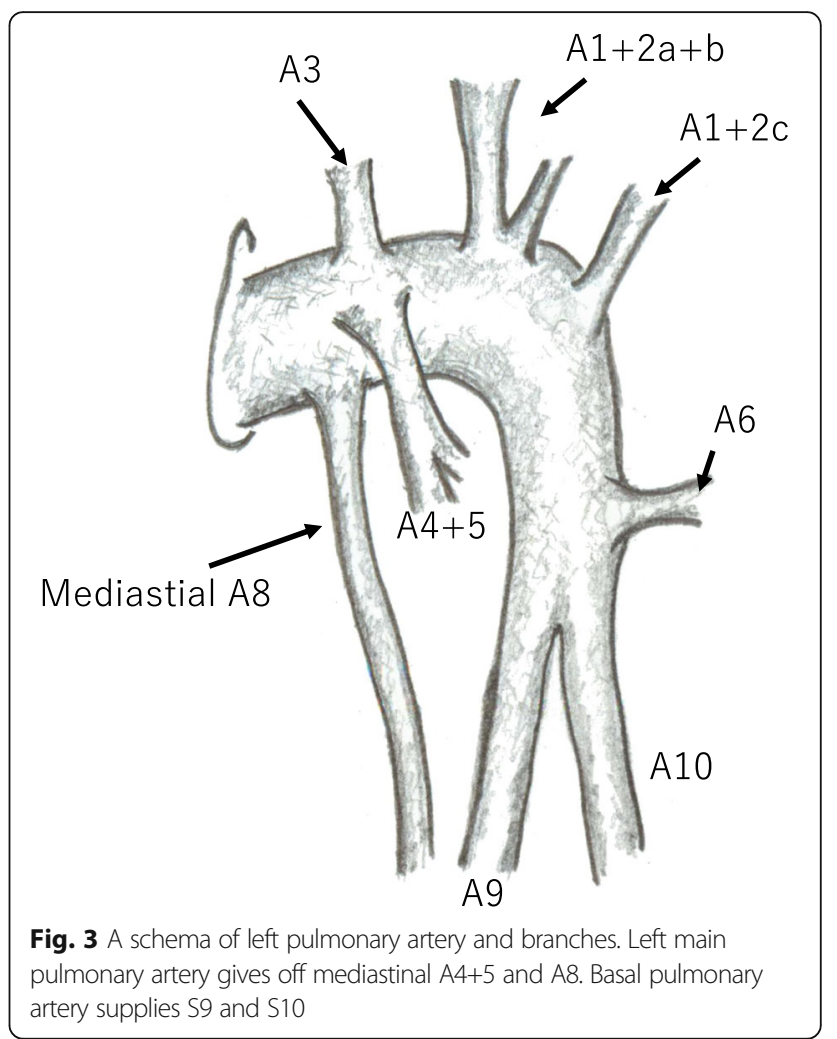

patterns have been reported, with mediastinal origin in $9.2 \%$, interlobar and mediastinal origin in $26.9 \%$, and interlobar origin in $63.9 \%$. Furthermore, 8 of 23 cases with mediastinal lingular artery were overlooked intraoperatively [2]. However, a branch from the first left pulmonary branch to the basal segment is rare.

It is possible that even the operator is not able to recognize a mediastinal lingular artery. Therefore, the possibility of missing a mediastinal basal lung artery is high. A total of 14 cases on the left side were reported only in Japan, including the present case (Table 1) [2-15].

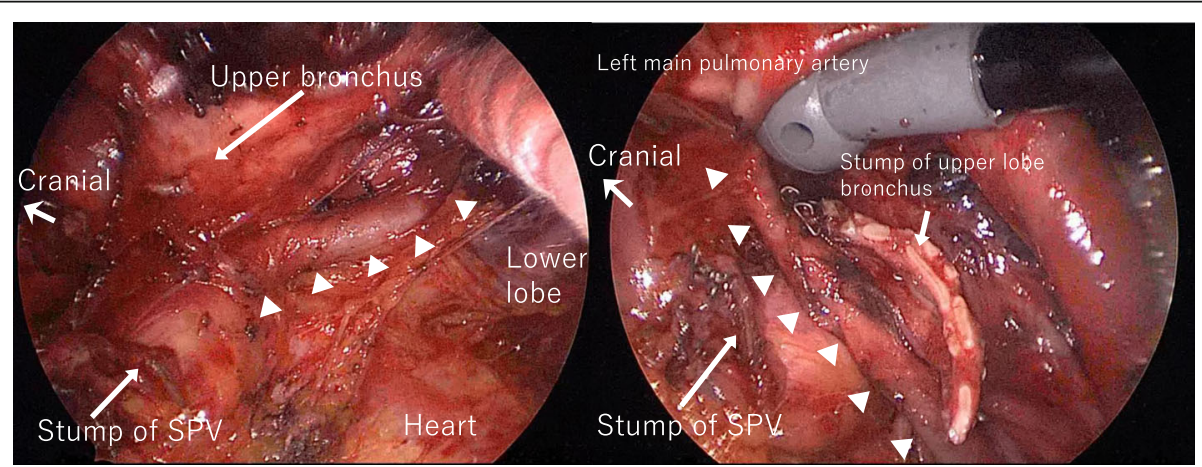

Fig. 2 Intraoperative findings. Pulmonary artery branch from the left main pulmonary artery to $\mathbf{S} 8$ is between the stump of the superior pulmonary vein (SPV) and the upper lobe bronchus (white arrowheads) 


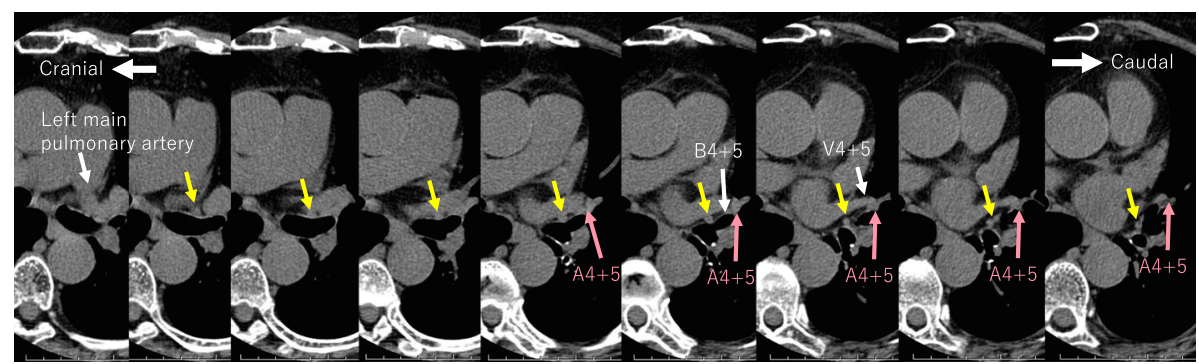

Fig. 4 Chest-computed tomography (mediastinal window setting). It shows a pulmonary branch from the left main pulmonary artery to S8 (yellow arrow). It is different from mediastinal A4+5 (pink arrow). It is between the left superior pulmonary vein and the left upper lobe bronchus

Table 1 Abnormal branch of the left pulmonary artery to the lower lobe: a review of the literature

\begin{tabular}{|c|c|c|c|c|c|}
\hline Report & & First author & Year & Preoperative modality & Preoperative diagnosis \\
\hline 1 & $A 9+10$ & Bamba & 1985 & Angiography & $\circ$ \\
\hline 2 & $\mathrm{~A} 5+8$ & Iwabuchi & 1995 & Contrast CT & $x$ \\
\hline 3 & $A 9+10$ & Sano & 1996 & Contrast CT & $x$ \\
\hline 4 & $A 8+9$ & Moriyama & 2009 & Contrast CT & $\circ$ \\
\hline 5 & $A 5+8+9+10$ & Kataoka & 2010 & Contrast CT & $\circ$ \\
\hline 6 & A8 & Sueda & 2011 & Contrast CT & $\circ$ \\
\hline 7 & A9 & Kaneda & 2012 & Contrast CT & $x$ \\
\hline 8 & $\mathrm{~A} 5+\mathrm{A} 8 \mathrm{~b}$ & Kozu & 2012 & Contrast CT & $x$ \\
\hline 9 & $A 8+9 b$ & Matsumoto & 2012 & $3 \mathrm{DCT}$ & $\circ$ \\
\hline 10 & A8 & Kato & 2014 & $3 \mathrm{DCT}$ & $x$ \\
\hline 11 & $\mathrm{~A} 4+5+9+10$ & Yajima & 2014 & Non-contrast $C T$ & $x$ \\
\hline 12 & $A 8 b+9 b+10$ & Kawai & 2015 & Contrast CT & $\circ$ \\
\hline 13 & $A 8+9+10$ & Sonoda & 2016 & 3DCT & $\circ$ \\
\hline 14 & $\mathrm{~A} 5+8+10$ & Nagata & 2016 & $3 \mathrm{DCT}$ & $\circ$ \\
\hline 15 & A8 & Our case & 2018 & Non-contrast $\subset T$ & $x$ \\
\hline
\end{tabular}

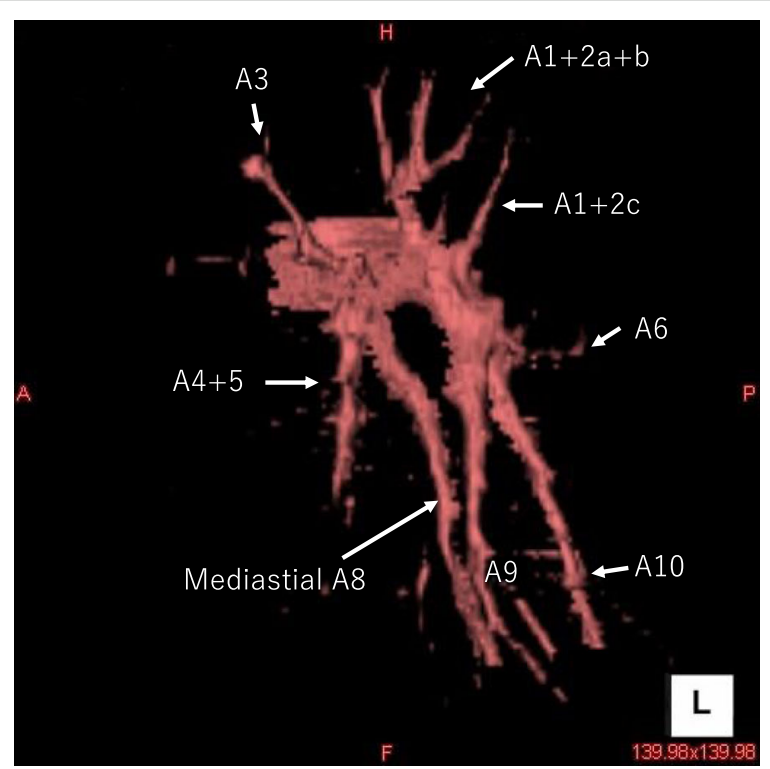

Fig. 5 Three-dimensional computed tomographic pulmonary angiography of our case without contrast medium. It was constructed by non-contrast $C$ retrospectively. It is easy to recognize left mediastinal A8 given off from main pulmonary artery 
It is possible to identify a basal lung artery preoperatively by contrast-enhanced CT or 3DCT. However, it has been reported that it was not diagnosed by non-contrast $\mathrm{CT}$. In the present case, the mediastinal basal lung artery was identified intraoperatively, though it was difficult to diagnose it preoperatively. Therefore, the discreet separation of the vessel and bronchus is very important. Retrospectively, CT showed the mediastinal basal artery, although the interpretation was difficult; careful CT reading is also important.

3DCT is useful to identify the running condition of the pulmonary vessels preoperatively. However, contrast medium should be avoided when there is renal dysfunction, allergy, and so on. On the other hand, 3DCT without contrast medium has been reported to have slightly poorer resolution but may be helpful [16]. Actually, we constructed 3DCT of our case using non-contrast CT retrospectively (Fig. 5). It was necessary to spend several hours to make this image because it was difficult to distinguish artery from vein without contrast medium. The surface of pulmonary artery may be jagged; however, it helps to comprehend pulmonary pattern of branching.

In conclusion, although preoperative identification of left pulmonary mediastinal branches was difficult on non-contrast $\mathrm{CT}$, a careful surgical procedure preserved the left pulmonary mediastinal A8.

\section{Abbreviations \\ 3DCT: Three-dimensional computed tomographic angiography; \\ CT: Computed tomography; FDG-PET: Fluorodeoxyglucose-positron emission tomography}

\section{Availability of data and materials}

Data sharing is not applicable to this article, as no datasets were generated or analyzed during the current study.

\section{Authors' contributions}

YM performed surgery, carried out the acquisition of data, and drafted the manuscript. NU and HA proofread the paper. All authors read and approved the final manuscript.

\section{Ethics approval and consent to participate}

Not applicable.

\section{Consent for publication}

The patient provided informed consent for the publishing.

\section{Competing interests}

The authors declare that they have no competing interests.

\section{Publisher's Note}

Springer Nature remains neutral with regard to jurisdictional claims in published maps and institutional affiliations.

Received: 9 April 2018 Accepted: 21 June 2018

Published online: 03 July 2018

\section{References}

1. Shields TW. Surgical anatomy of the lungs. In: Shields TW, LoCicero 3rd J, Reed CE, Feins RH, editors. General thoracic surgery. 7th ed. Philadelphia: Lippincott Williams \& Wilkins; 2009. p. 79.
2. Nagata T, Nakamura Y, Kariatsumari K, Otsuka T, Aoki M, Sato M. Potentially overlooked branches of the left pulmonary artery. Asian Cardiovasc Thorac Ann. 2016;24:676-80.

3. Bamba J, Tomoyasu H, Tanimura S, Masaki M. Abnormal branching of the left pulmonary artery to the lateral and posterior basal segments. Nihon Kyobu Geka Gakkai Zasshi. 1985;33:366-8.

4. Iwabuchi S, Okabe T, Koike K. Aberrant ramuts of left pulmonary artery to inferior lingular and anterior basal segment (A5+8). Jpn J Chest Surg. 1995;9:159-63.

5. Sano M, Mizuno T, lizuka M, Yamada T, Kasugai T, Ishiguro H. Abnormal branching of left pulmonary artery to the lateral and posterior basal segments. Nihon Kyobu Geka Gakkai Zasshi. 1996;44:1772-5.

6. Moriyama S, Miyoshi K, Tada A, Kurosaki T. A case report of abnormal branching of left A8+9 pulmonary artery. Jpn J Chest Surg. 2009;23:58-61.

7. Kataoka K, Nishikawa T, Fujiwara T, Matsuura M. A case of lung cancer with an extremely rare branching pattern of the left $A 5+8+9+10$ pulmonary artery. Haigan. 2010;50:362-5.

8. Sueda T, Takami K, Omiya H, Kodama Y, Kuriyama K, Tsujinaka T. A case of abnormal branching of the left anterobasal pulmonary artery with primary lung cancer. J Jpn Surg Assoc. 2011;72:630-5.

9. Kaneda Y, Samura M, Kanayama Y, Okazaki M, Noshima S, Zempo N. A case of abnormal branching of the left A9 pulmonary artery from the left main pulmonary artery. J Jpn Surg Assoc. 2012;73:1920-3.

10. Kozu Y, Maniwa T, Isaka M, Ohde $Y$, Kondo H, Endo M. A case of abnormal branching of $\mathrm{A} 5$ and $\mathrm{A} 8 \mathrm{~b}$ as a common trunk from the left main pulmonary artery. Jpn J Chest Surg. 2012;26:772-5.

11. Matsumoto K, Yamasaki N, Tsuchiya T, Miyazaki T, Tomoshige K, Hayashi H, et al. Three-dimensional computed tomography for a mediastinal basal pulmonary artery. Ann Thorac Surg. 2012;94:e115-6.

12. Kato $H$, Oizumi $H$, Inoue $T$, Watarai $H$. A case report of lung cancer with abnormal branching of the left A8 pulmonary artery detected during left upper lobectomy. Haigan. 2014;54:6-11.

13. Yajima $K$, Neyatani $H$, Takahashi T. A resected case of left upper lung cancer with $\mathrm{A} 4, \mathrm{~A} 5, \mathrm{~A} 9$ and $\mathrm{A} 10$ arising as a common trunk from the left main pulmonary artery. Haigan. 2014;54:84-8

14. Kawai N, Kawaguchi T, Yasukawa M, Tojo T. Two cases of lower lobectomy with mediastinal inferior lobar branch in lung cancer. Jpn J Chest Surg. 2015;29:786-91.

15. Sonoda D, Mikubo M, Shiomi K, Satoh Y. Abnormal branching of the left lingular pulmonary artery diagnosed by three-dimensional computed tomographic angiography in a patient with lung cancer: a case report. J Cardiothorac Surg. 2016;11:17.

16. Watanabe S, Arai K, Watanabe T, Koda W, Urayama H. Use of threedimensional computed tomographic angiography of pulmonary vessels for lung resections. Ann Thorac Surg. 2003;75:388-92.

\section{Submit your manuscript to a SpringerOpen ${ }^{\circ}$ journal and benefit from:}

- Convenient online submission

Rigorous peer review

- Open access: articles freely available online

- High visibility within the field

Retaining the copyright to your article

Submit your next manuscript at $>$ springeropen.com 